\title{
An Improved Artificial Colony Algorithm Model for Forecasting Chinese Electricity Consumption and Analyzing Effect Mechanism
}

\author{
Jingmin Wang, Jian Zhang, and Jing Nie \\ Department of Business Administration, North China Electric Power University, Baoding 071000, China \\ Correspondence should be addressed to Jian Zhang; zj_ncepu_sub@163.com
}

Received 22 March 2016; Revised 27 June 2016; Accepted 20 July 2016

Academic Editor: Marek Lefik

Copyright (C) 2016 Jingmin Wang et al. This is an open access article distributed under the Creative Commons Attribution License, which permits unrestricted use, distribution, and reproduction in any medium, provided the original work is properly cited.

\begin{abstract}
Electricity consumption forecast is perceived to be a growing hot topic in such a situation that China's economy has entered a period of new normal and the demand of electric power has slowed down. Therefore, exploring Chinese electricity consumption influence mechanism and forecasting electricity consumption are crucial to formulate electrical energy plan scientifically and guarantee the sustainable economic and social development. Research has identified medium and long term electricity consumption forecast as a difficult study influenced by various factors. This paper proposed an improved Artificial Bee Colony (ABC) algorithm which combined with multivariate linear regression (MLR) for exploring the influencing mechanism of various factors on Chinese electricity consumption and forecasting electricity consumption in the future. The results indicated that the improved $\mathrm{ABC}$ algorithm in view of the various factors is superior to traditional models just considering unilateralism in accuracy and persuasion. The overall findings cast light on this model which provides a new scientific and effective way to forecast the medium and long term electricity consumption.
\end{abstract}

\section{Introduction}

With the development of economy and technology, particularly in thirty years or so of reform and opening-up, Chinese electricity consumption achieves a sustained and rapid development. However, as illustrated in Figure 1, along with the economic development entering the "new normal," the national economy is in a period of transition from rapid growth to steady growth and the electricity consumption has been fluctuating.

Starting in 2012, on account of the decline in electricity consumption proportion of high energy-intensive industries, the growth of electricity demand in power industry has slowed down significantly. Pumping capital into installed capacity in early time brought about relative surplus buyer's market and the buying-market has gradually formed in recent years $[1,2]$. Nevertheless, electric power replacement, as one of the major initiatives to realize energy consumption transition and solve environmental pollution problems in the current situation, is conducive to increase the proportion of electricity in the energy consumption. As a result, facing the new normal and power supply and demand contradiction, it is important to explore Chinese electricity consumption influence mechanism and forecast electricity consumption which is significant for scientifically formulating electrical energy plan and guaranteeing the sustainable economic and social development [3-5].

At present, studies on forecasting medium and long term electricity consumption have proposed multiple methods which contain traditional linear regression, time series and emerging grey prediction [6-10], and so forth. While these methods only rely on electricity consumption data to predict the future and ignore the various factors closely related, actually, electricity consumption is relevant to numerous multidimensional and dynamically changing factors and some correlation may exist between them. The parameter set of higher complexity is necessary to express the changing tendency under the impact of these factors. 


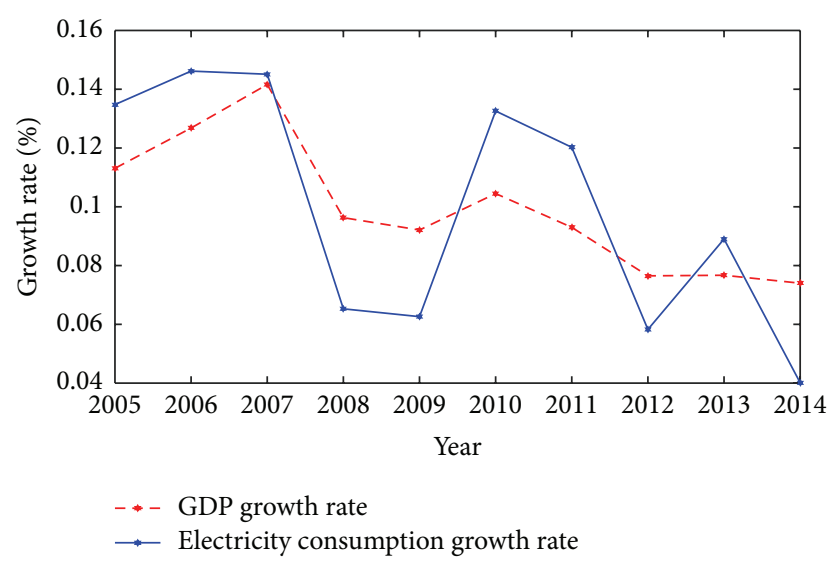

FIGURE 1: GDP growth rate and electricity consumption growth rate.

What is more, it is improper to use Artificial Neural Network (ANN) and Kalman Filtering for medium and long term electricity consumption forecast in years, because these methods usually need large amounts of data $[11,12]$. And they could not show the relationship between each influencing factor and electricity consumption excellently or provide a good guidance for electrical energy plan.

Combined with the feedback at home or abroad, the historical situation of Chinese electricity use, and the change in demand of "new normal" electricity, this paper puts forward the main influence mechanism between electricity consumption and the variables from economy, society, industry, and environment. Thereby, an improved ABC method is suggested to forecast electricity consumption.

This study will make a significant contribution that will be embodied in the following two aspects: firstly, this study provides a full and objective analysis of influence mechanism between the electricity consumption and the variables from economy, society, industry, and environment and elucidates the relationship between them by mathematical statistics method based on a large amount of literature data and the actual situation of Chinese development; secondly, an improved $\mathrm{ABC}$ method was used to forecast electricity consumption by creating intended model. Given the example calculation, the model presented herein can assess the effect of various factors on the electricity consumption clearly and confer further advantages on medium and long term forecasting. In the field of prediction, many scholars regard ABC method as an auxiliary algorithm for numerical optimization, and little attention has been focused on a direct use of the advantages of the $\mathrm{ABC}$ method to build a model to predict medium and long term electricity consumption in the last years. Taken together, our studies have certain innovation and application value.

This paper is divided into six sections. After a brief introduction, Section 2 presents the influence factors and forecasting methods of electricity consumption. Section 3 describes the data in detail and Section 4 presents the ABC algorithm and the improvement in this paper. Numerical results which are then discussed are described in Section 5. The conclusions of the paper are summarized in Section 6.

\section{Literature Review}

Electricity, as a special commodity which has difficulty in storing, has to be fully prepared for the forecast, cohesion, and balance of total supply and demand. And as a result, a healthy grid situation will be manifest that the reasonable demand will be met by scientific supply and the problem of regional excess and overall energy shortage will be gradually solved. The objective of accurate modeling of the electricity consumption calls for attention to a few extremely important points. The first point is to discern all of the necessary variables that are bound up with electricity consumption in a given area [13]; the second point is to choose a suitable modeling methodology according to the characteristics of the electricity consumption that can handle the difficulties of the consumption modeling task and obtain the accurate result.

\subsection{The Literature of the Influence Factors of Electricity} Consumption. On account of the effect of socioeconomic development and the changes in policy environment, the factors which could act on electricity consumption tend to be nonlinear and nonstationary and it is conceivable that the relationship between the input variables and the output variable is undiscovered [14-16].

Previous studies have established that many different variables have been used to model the electricity consumption. Table 1 presents a brief summary of some of the important recent studies on the field.

The first aspect is to ascertain a causal relationship between the economic factors and electricity consumption. Along with economic growth, the productivity and living reveal a growing reliance on electricity demand, and, more than that, the interaction between electricity consumption and economy growth is becoming more and more obvious. In the light of most theories and empirical researches, the economic development which regards real GDP, fixed assets investment, and foreign direct investment as the test variables is central to electricity consumption.

In a study undertaken by Karanfil and Li [17], it was shown that the relationship between electricity consumption and economic growth was affected at the national income level, geographic location, development level, and other factors considering the power dependence and urbanization with the panel data of 160 countries from 1980 to 2010. Ciarreta and Zarraga have demonstrated that the strong causality from electricity consumption to real GDP has characteristics of unidirectional and negative in the perspective of dynamic during a certain time period [18]. Bianco et al. [19] developed linear regression models using historical electricity consumption, gross domestic product (GDP), GDP per capita, and population and the models showed annual electricity consumption was strongly related to the selected variables. Moreover, their time sequence would maintain a state of relative balance in the long term. Tang et al. [20] have highlighted that electricity consumption and economic growth in Portugal have a bidirectional causality from a long term point of view adopting boundary detection method, Granger causality test based on vector error correction model, and other statistical methods. Polemis and Dagoumas 


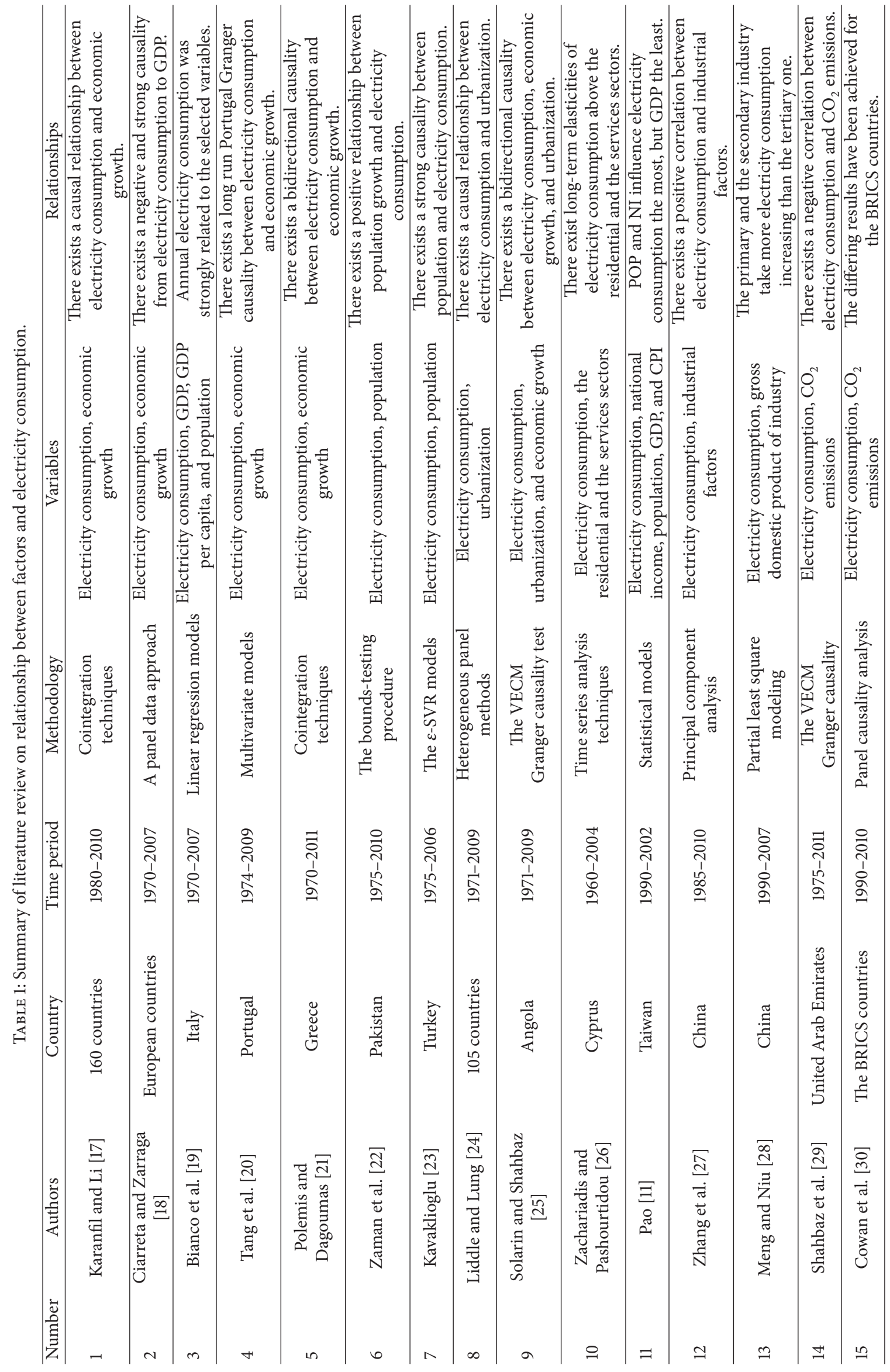


[21] using the same statistical methods have indicated the causal correlation between electricity consumption and economic growth in the case of Greece is bidirectional. Especially in the past ten years, a relatively strong impact is performed from the electricity consumption to GDP.

The second aspect takes the social factors that affect the electricity consumption into consideration. With regard to the development of any country, the social factors are thought to be prerequisite and impact on the development of economy and industry directly. Social factors mainly contain three aspects; they are population, urbanization level, and people living standard. For electricity consumption, it is a core problem whether the growth of electricity consumption can match the trend of population, economic, and social development [22]. And one can even say the social changes in all aspects will make a far-reaching difference in electricity sales market in the future.

Before Kavaklioglu [23] conducted a study of electricity consumption forecast in Turkey utilizing the $\varepsilon$-SVR models, he obtained that the electricity consumption would rise accordingly as the growth of the population allowing for the influence factors including GNP, population, imports, and exports. Collecting urbanization level and other related factors index data of 105 countries from 1971 to 2009, Liddle and Lung [24] clearly pointed out that the improvement of urbanization level would bring the increase in electricity consumption largely based on heterogeneous panel methods; nevertheless the connection found that the relationship between urbanization level and electricity consumption was not a simple linear relationship. In both Solarin and Zachariadis investigations on the correlativity between economic growth, urbanization, and electricity consumption, the findings raised the same conclusion that electricity consumption not only with economic growth but also with urbanization level is in a significant causal relationship in the long term $[25,26]$. Another study aimed at the interrelation of electricity consumption and consumer price index (CPI) certified CPI has an effect on electricity consumption in Taiwan [11]. The electricity price was involved in some discussions while as for China it is not an effective index by reason that the price of Chinese electricity consumption is fixed and rarely changed.

The third aspect focuses on industrial factors. Industrial structure refers to the structure of a country or a region's economic industry and their mutual relations. Researches could measure a country or a region's industrial structure from multiple angles, such as the output value structure, labor structure, and relative labor productivity.

Regarding industrial factors including industrial output value, commodity exports, and added services industry value, Zhang et al. [27] utilized principal component analysis and carried on the analysis to show the existence of the positive correlativity between electricity consumption and industrial factors. Meng and Niu [28] have stated that the relationship between electricity consumption and its factors was not as complexity as imagined, so that it was not complicated to achieve the relationship between them using multivariate regression model. Through partial least square modeling, the results of variables importance analysis were elucidated that the secondary industry took more electricity consumption increasing than the primary and the tertiary one [31]. Therefore, most of the studies chose the secondary industry contribution to the GDP as the main indicator measuring the industrial structure during the period.

The fourth aspect is with respect to the environmental factors. In the process of construction of low-carbon society, electricity development plays an important role. Practice has proven that greenhouse gas emissions have been the top in all industries during the link of electricity production which is predominantly coal-fired. Meanwhile, the emissions during the link of using electricity are less than the emissions of other primary energy (mainly including coal, oil, and natural gas) for the environment. Hence, the mutual influence mechanism has existed distinctly between electricity consumption and environmental factors.

The result of Shahbaz et al.s study pointed out a negative correlation relationship and a back donation between electricity consumption and carbon emissions in United Arab Emirates [29]. In allusion to the different situations of the BRICS countries, Cowan et al. [30] verified that electricity consumption was the Granger causality of $\mathrm{CO}_{2}$ emissions in India, while there was no Granger causality between electricity consumption and $\mathrm{CO}_{2}$ emissions in Brazil, Russia, China, and South Africa.

The summary of literature review on relationship between factors and electricity consumption is shown in Table 1.

\subsection{The Literature of Forecasting Methods regarding Electricity} Consumption. The common methods among the research studies on electricity consumption forecast derive from traditional consumption method, elasticity coefficient method, regression analysis, gray prediction method, Support Vector Machine (SVM), and then modern intelligent algorithms, such as Neural Network, and Wavelet Analysis method, and other meta-heuristics, such as Genetic Algorithms and Particle Swarm Optimization. In addition, prediction accuracies have discrepancies owing to different methods.

At present, the scientific research strength of medium and long term electricity consumption forecast has less adequacy than short term load forecast technology because of its more impact factors, the small amount of historical data, and having more difficulties in making accurate quantitative research.

Via the statistical analysis to determine the relationship between variables, the traditional forecast method, such as grey prediction and regression analysis, shows a good fitting capacity whereas the forecast results cause big error in the future changing trend. Fitting degree and prediction precision perform unsatisfactorily although many scholars use some combination methods to ameliorate the accuracy especially when the model has many variables [32-38]. SVM method can better deal with the nonlinearity and uncertainty among factors influencing the long term power consumption, but it still has some risks appearing local minimum value [39].

So far, some intelligence algorithms, such as ANN, Genetic Algorithm (GA), and Ant Colony Optimization, have received particular attention in estimating electricity consumption and especially in short term electricity 
consumption forecasting since they can handle current data to an arbitrary degree of accuracy $[40,41]$.

Through the comparison with conventional regression model, Azadeh et al. showed the estimation of Iran electricity consumption done by GA is more fitted than regression method [42]. They also used an integrated GA and ANN to estimate and predict electricity consumption while the relative error of the method was small [43]. Kiran et al. verified the results of forecasting electricity consumption in Turkey by ABC and PSO techniques outperformed the results forecasted by Ant Colony Optimization [44]. Azadeh et al. compared three meta-heuristics, namely, GAs, Artificial Immune System (AIS), and Particle Swarm Optimization (PSO), with each other in estimation of electricity consumption in the selected countries. With fitted random variables, AIS method with the Clonal Selection Algorithm shows satisfactory results and has been selected as the preferred method [45].

Some of these algorithms require large amounts of historical load data processing to let the computer learning mapping relationships predict the future of the load. Some have strong randomness and fall into local optimum. Modeling the consumption carried out with these algorithms has strong robustness and nonlinear mapping ability to solve current data, but they are not good for prediction since they do not use any mathematical models and present a slow learning convergence speed and a weak generalization ability.

In consequence, with the purpose of exhibiting good global optimization and robustness characteristics as well as accuracy in prediction, it is necessary to build a forecast model under the support of mathematical theory coalescing both mathematical analysis methods and intelligent algorithms.

\section{Data Source}

This study is based on annual data of electricity consumption, real gross domestic product (GDP), fixed asset investment (FAI), foreign direct investment (FDI), population, urbanization level, household consumption level, real GDP of secondary industry, and carbon emission covering a time period from 1990 to 2014 in China. The time series data are collected from International Monetary Fund Data and Statistics (IMF 2015) and World Bank, world development indicators (WDI, 2015). The real GDP series are transformed from the nominal GDP in constant 1990 prices. These factors selection is based on the above literature analysis and Chinese actual conditions.

In the process of modeling and predicting of electricity consumption, the real GDP and fixed assets investment need to be deemed as important factors into consideration. Although there is no strict sense of the causality between electricity consumption and economic growth in China, electricity consumption as a reflection of the power industry development in the economic system can ahow a country or a region's economic operation. Meanwhile FDI is thought to act as a vital economic factor in view of the advancement of energy internet globalization.
Population and urbanization level, as important factors, should be taken into account in the study as well. There is explanation of urbanization level that it is generally represented with the proportion of urban population accounting for total population. And it is exceedingly significant index to measure economic development of a country or area. In accelerating the process of urbanization, it brings a series of electrification development of the society, which will lead to increasing power consumption, and increasing population will also pull the economic development and growth in electricity consumption sequentially.

It can give no cause for much criticism of the interaction between electricity consumption and industrial structure. The adjustment of the industry is an important cause of promoting economic development and fluctuating of electricity consumption due to the different electricity consumption per unit GDP of industrial sectors.

Apart from that, carbon emission is supposed to be an essential factor in the study of electricity consumption in virtue of a strategy of low carbon energy development via improving the power conversion efficiency and realizing the energy alternative which build electricity as the core.

Table 2 shows the primary data of electricity consumption and the factors mentioned before.

On account of different numerical size and dimension of various factors, this paper applies the method of $Z$-Score to normalizing them according to the annual order. The process is as follows:

$$
X_{i}^{\prime}=\frac{\left(X_{i}-\bar{X}\right)}{S_{i}}
$$

$X_{i}$ and $X_{i}^{\prime}$ represent primary data and normalized data, respectively, $\bar{X}$ is the average value, and $S_{i}$ is the standard deviation. The values of these standardized variables fluctuate around zero. Greater than zero is above the average and less than zero is under the average. The normalized data is shown in Table 3.

In order to find some relationship between electricity consumption and various factors, the contrast of change trend of these variables is illustrated by Figure 2 .

In Figure 2, we can see the change trend of electricity consumption is same to these factors. And we can trust there must be some relationship between electricity consumption and these factors. Moreover, correlation analysis of the factors and electricity consumption also show the intimate connection between them. Because the counting process is subordinate, the results of correlation analysis are exhibited in Supplementary Material, available online at http://dx.doi.org/ $10.1155 / 2016 / 8496971$.

\section{Methodological Framework}

4.1. Introduction of $A B C$ Algorithm. Artificial Bee Colony $(\mathrm{ABC})$ algorithm, as a swarm intelligence optimization algorithm, has a superior convergence speed on the basis of the intelligent behavior of honey bees. The main characteristic of $\mathrm{ABC}$ algorithm is that it only needs to contrast the advantages or disadvantages with other solutions rather than 
TABLE 2: Primary data of various factors and electricity consumption.

\begin{tabular}{|c|c|c|c|c|c|c|c|c|c|}
\hline Year & $\begin{array}{c}\text { Real } \\
\text { GDP } \\
\text { (billion } \\
\text { RMB) }\end{array}$ & $\begin{array}{c}\text { FAI } \\
\text { (billion } \\
\text { RMB) }\end{array}$ & $\begin{array}{c}\text { FDI } \\
\text { (million } \\
\text { dollars) }\end{array}$ & $\begin{array}{l}\text { Population } \\
\text { (million } \\
\text { people) }\end{array}$ & $\begin{array}{l}\text { Urbanization } \\
\text { level (\%) }\end{array}$ & $\begin{array}{l}\text { Household } \\
\text { consumption } \\
\text { level } \\
(\mathrm{RMB})\end{array}$ & $\begin{array}{l}\text { Real GDP of } \\
\text { secondary } \\
\text { industry } \\
\text { (billion } \\
\text { RMB) }\end{array}$ & $\begin{array}{c}\text { Carbon } \\
\text { emission } \\
\text { (million tons) }\end{array}$ & $\begin{array}{c}\text { Electricity } \\
\text { consumption } \\
\text { (billion } \\
\mathrm{kW} \cdot \mathrm{h} \text { ) }\end{array}$ \\
\hline 1990 & 1882.5 & 451.7 & 10289 & 1143 & 0.2641 & 1510 & 743 & 2458 & 623 \\
\hline 1991 & 1954.8 & 559.5 & 11550 & 1158 & 0.2694 & 1701 & 1252 & 2591 & 680 \\
\hline 1992 & 2134.2 & 808.0 & 19202 & 1172 & 0.2746 & 2027 & 1481 & 2723 & 759 \\
\hline 1993 & 2438.1 & 1307.2 & 38955 & 1185 & 0.2799 & 2577 & 1719 & 2877 & 843 \\
\hline 1994 & 2778.5 & 1704.2 & 43206 & 1199 & 0.2851 & 3496 & 2001 & 3029 & 926 \\
\hline 1995 & 3141.9 & 2001.9 & 48133 & 1211 & 0.2904 & 4283 & 2104 & 3228 & 1002 \\
\hline 1996 & 3485.0 & 2291.4 & 54804 & 1224 & 0.3048 & 4839 & 2290 & 3323 & 1076 \\
\hline 1997 & 3833.9 & 2494.1 & 64408 & 1236 & 0.3191 & 5160 & 2372 & 3314 & 1128 \\
\hline 1998 & 4190.4 & 2840.6 & 58557 & 1248 & 0.3335 & 5425 & 2588 & 3312 & 1160 \\
\hline 1999 & 4518.5 & 2985.5 & 52659 & 1258 & 0.3478 & 5854 & 2650 & 3423 & 1231 \\
\hline 2000 & 4862.8 & 3291.8 & 59360 & 1267 & 0.3622 & 6280 & 3010 & 3514 & 1347 \\
\hline 2001 & 5272.8 & 3721.3 & 49670 & 1276 & 0.3766 & 6860 & 2531 & 3674 & 1463 \\
\hline 2002 & 5710.4 & 4350.0 & 55010 & 1285 & 0.3909 & 7703 & 2940 & 4025 & 1647 \\
\hline 2003 & 6228.9 & 5556.7 & 56140 & 1292 & 0.4053 & 8472 & 3807 & 4723 & 1903 \\
\hline 2004 & 6853.7 & 7047.7 & 64072 & 1300 & 0.4176 & 9422 & 3742 & 5521 & 2197 \\
\hline 2005 & 7545.2 & 8877.4 & 63805 & 1308 & 0.4299 & 10493 & 4052 & 6326 & 2494 \\
\hline 2006 & 8398.6 & 10999.8 & 67080 & 1314 & 0.4434 & 11760 & 4493 & 6926 & 2859 \\
\hline 2007 & 9463.5 & 13732.4 & 78340 & 1321 & 0.4589 & 13786 & 5171 & 7518 & 3271 \\
\hline 2008 & 10803.5 & 17282.8 & 95253 & 1328 & 0.4699 & 15781 & 5499 & 7663 & 3438 \\
\hline 2009 & 11843.9 & 22459.9 & 91804 & 1335 & 0.4834 & 17175 & 6439 & 8037 & 3643 \\
\hline 2010 & 12934.8 & 25168.4 & 108820 & 1341 & 0.4995 & 19109 & 7849 & 8472 & 4192 \\
\hline 2011 & 14286.4 & 31148.5 & 117698 & 1347 & 0.5127 & 21810 & 7738 & 9206 & 4693 \\
\hline 2012 & 15615.1 & 37469.5 & 113294 & 1354 & 0.5257 & 24565 & 7982 & 9415 & 4976 \\
\hline 2013 & 16809.6 & 44629.4 & 118721 & 1361 & 0.5373 & 26955 & 8368 & 9674 & 5322 \\
\hline 2014 & 18098.9 & 51276.1 & 119705 & 1368 & 0.5477 & 28844 & 8811 & 9761 & 5523 \\
\hline
\end{tabular}

comprehending the special information of the problem. Gradually, the global optimal value will emerge through the local optimization of each individual worker bee.

However, many intelligent algorithms have been brought forward in recent decades such as Particle Swarm Optimization (PSO) and Artificial Neural Network (ANN) algorithm and Genetic Algorithm (GA). In many areas, it cannot be denied that these algorithms have solved a great deal of problems and made difference [46-51]. But in this paper, these algorithms could be not so appropriate. Obviously, the most important reason is that the data quantity is too lacking to use these intelligent algorithms and it could lead to a huge error which does not want to be seen. Fortunately, the initial purpose of putting forward $\mathrm{ABC}$ algorithm is to solve the optimization problem of multivariate function [52]. And Akay and Karaboga analyzed the size of the population for $\mathrm{ABC}$ algorithm and drew a conclusion that $\mathrm{ABC}$ algorithm did not have to use a big value of colony size to solve optimization problems [53]. Just because of these, the improved $\mathrm{ABC}$ algorithm is believed to solve the problem in the field of electricity consumption although this is the first attempt.

4.2. Traditional Algorithm Procedure of $A B C$. In ABC algorithm, the swarm is composed of employed bees, onlookers, and scouts and the location of a food source is abstracted into a point of $D$-dimensional space. The process of finding the optimal food source is the procedure of searching for the optimal solution. The position of the swarm represents the solution of optimization problem and the benefit of the source symbolizes the adaptive value of the optimization problem.

The bees search all of the food sources recurrently and location of food source is denoted with a $D$-dimensional vector quantity:

$$
X_{j}=\left(x_{1}, x_{2}, \ldots, x_{D}\right), \quad j \in[1, D]
$$


TABLE 3: Normalized data of various factors and electricity consumption.

\begin{tabular}{|c|c|c|c|c|c|c|c|c|c|}
\hline Year & $\begin{array}{l}\text { Real } \\
\text { GDP }\end{array}$ & FAI & FDI & Population & $\begin{array}{c}\text { Urbanization } \\
\text { level }\end{array}$ & $\begin{array}{c}\text { Household } \\
\text { consumption } \\
\text { level } \\
\end{array}$ & $\begin{array}{c}\text { Real GDP of } \\
\text { secondary } \\
\text { industry }\end{array}$ & $\begin{array}{l}\text { Carbon } \\
\text { emission }\end{array}$ & $\begin{array}{c}\text { Electricity } \\
\text { consumption }\end{array}$ \\
\hline 1990 & -1.11 & -0.78 & -1.75 & -1.93 & -1.41 & -1.12 & -1.34 & -1.12 & -1.07 \\
\hline 1991 & -1.08 & -0.78 & -1.71 & -1.71 & -1.34 & -1.10 & -1.13 & -1.07 & -1.04 \\
\hline 1992 & -1.03 & -0.76 & -1.47 & -1.51 & -1.27 & -1.05 & -1.04 & -1.02 & -0.99 \\
\hline 1993 & -0.97 & -0.74 & -0.85 & -1.31 & -1.20 & -0.96 & -0.95 & -0.96 & -0.93 \\
\hline 1994 & -0.90 & -0.71 & -0.72 & -1.11 & -1.13 & -0.90 & -0.83 & -0.90 & -0.88 \\
\hline 1995 & -0.84 & -0.68 & -0.57 & -0.92 & -1.06 & -0.86 & -0.79 & -0.83 & -0.83 \\
\hline 1996 & -0.77 & -0.66 & -0.36 & -0.73 & -0.92 & -0.79 & -0.72 & -0.79 & -0.79 \\
\hline 1997 & -0.71 & -0.65 & -0.06 & -0.55 & -0.77 & -0.70 & -0.68 & -0.80 & -0.76 \\
\hline 1998 & -0.65 & -0.62 & -0.24 & -0.38 & -0.62 & -0.66 & -0.60 & -0.80 & -0.74 \\
\hline 1999 & -0.59 & -0.61 & -0.43 & -0.23 & -0.47 & -0.60 & -0.57 & -0.75 & -0.69 \\
\hline 2000 & -0.51 & -0.59 & -0.22 & -0.09 & -0.33 & -0.51 & -0.43 & -0.72 & -0.62 \\
\hline 2001 & -0.44 & -0.56 & -0.52 & 0.05 & -0.18 & -0.38 & -0.62 & -0.66 & -0.55 \\
\hline 2002 & -0.34 & -0.52 & -0.35 & 0.17 & -0.03 & -0.30 & -0.45 & -0.52 & -0.43 \\
\hline 2003 & -0.23 & -0.44 & -0.32 & 0.28 & 0.12 & -0.18 & -0.10 & -0.26 & -0.27 \\
\hline 2004 & -0.10 & -0.36 & -0.07 & 0.40 & 0.25 & -0.09 & -0.13 & 0.05 & -0.09 \\
\hline 2005 & 0.05 & -0.22 & -0.08 & 0.51 & 0.38 & 0.02 & -0.01 & 0.36 & 0.10 \\
\hline 2006 & 0.24 & -0.07 & 0.02 & 0.61 & 0.53 & 0.19 & 0.17 & 0.59 & 0.33 \\
\hline 2007 & 0.49 & 0.09 & 0.37 & 0.71 & 0.69 & 0.35 & 0.45 & 0.82 & 0.58 \\
\hline 2008 & 0.67 & 0.27 & 0.90 & 0.81 & 0.81 & 0.61 & 0.58 & 0.87 & 0.69 \\
\hline 2009 & 0.87 & 0.76 & 0.79 & 0.91 & 0.95 & 0.81 & 0.96 & 1.01 & 0.82 \\
\hline 2010 & 1.12 & 0.84 & 1.32 & 1.01 & 1.12 & 1.05 & 1.53 & 1.18 & 1.16 \\
\hline 2011 & 1.37 & 1.18 & 1.59 & 1.10 & 1.27 & 1.32 & 1.48 & 1.46 & 1.47 \\
\hline 2012 & 1.58 & 1.72 & 1.46 & 1.20 & 1.41 & 1.65 & 1.58 & 1.54 & 1.65 \\
\hline 2013 & 1.82 & 2.22 & 1.63 & 1.30 & 1.54 & 1.96 & 1.73 & 1.64 & 1.87 \\
\hline 2014 & 2.06 & 2.67 & 1.66 & 1.41 & 1.67 & 2.22 & 1.91 & 1.68 & 1.99 \\
\hline
\end{tabular}

The formula of determining the initial solution is

$$
\begin{aligned}
& x_{i j}=x_{\min , j}+\operatorname{rand}(0,1)\left(x_{\max , j}-x_{\min , j}\right), \\
& \qquad j \in[1, D], i \in[1, S N] .
\end{aligned}
$$

$C$ and $S N$ are the cycle index and the number of honey bees, respectively. The bees select the food source in accordance with the roulette wheel. Fitness $s_{i}$ is the adaptation degree:

$$
\text { fitness }_{i}= \begin{cases}\frac{1}{1+f\left(X_{i}\right)} & f\left(X_{i}\right) \geq 0 \\ 1+\left|f\left(X_{i}\right)\right| & f\left(X_{i}\right)<0 .\end{cases}
$$

$P_{i}$, the selected probability, is

$$
P_{i}=\frac{\text { fitness }_{i}}{\sum_{j=1}^{F N} \text { fitness }_{j}} .
$$

The bigger value of $P_{i}$ means the better nectar source and then the better nectar source will gather more scouts. So the onlookers could find the best nectar source. The search formula of onlookers updating the location of nectar source by employed bees is

$$
\begin{aligned}
& v_{i j}=x_{i j}+r_{i j}\left(x_{i j}-x_{k j}\right), \\
& \qquad j \neq k, j \in[1, D], i \in[1, S N] .
\end{aligned}
$$

Hereinto, $k \in(1,2,3, \ldots, S N), j \in\{1,2, \ldots, D\}, r_{i j} \in[1$, 1]. However, this method also has disadvantages such as dealing with multiobjective or multiextreme problems. Consequently, it is always used to optimize the parameters and thresholds in other algorithms as an auxiliary method which can reduce the training time [54].

4.3. ABC Model in This Paper. This paper suggests that influence factors and electricity consumption are regarded as independent variables $\left(A=a_{1}, a_{2}, a_{3}, \ldots, a_{D}\right)$ and dependent variable separately. In the previous studies, Bianco et al. [19] and Meng and Niu [28] both have developed linear regression models to forecast electricity consumption and the models showed an outstanding outcome. So there is linear function between the variables:

$$
\mathrm{Tec}=f\left(a_{1}, a_{2}, a_{3}, \ldots, a_{D}\right) .
$$




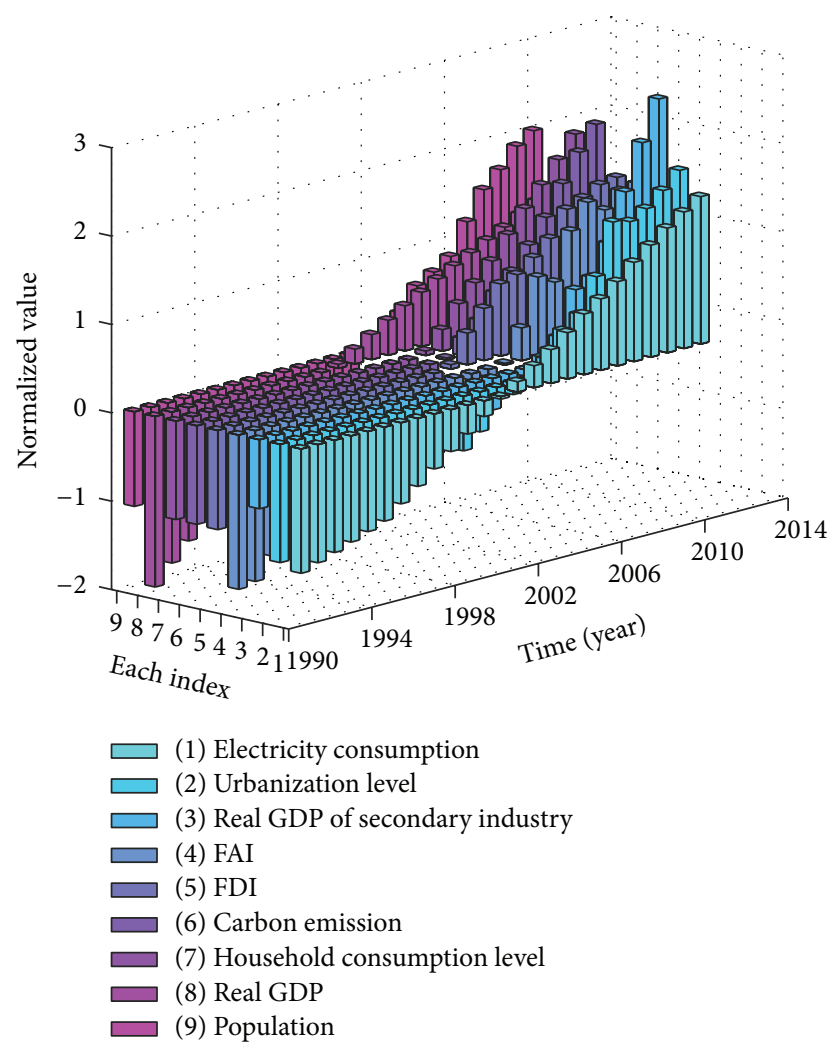

FIGURE 2: The contrast of change trend between electricity consumption and various factors.

This can also be written as follows:

$$
a_{1} x_{1}+a_{2} x_{2}+a_{3} x_{3}+\cdots+a_{D} x_{D}+x_{D+1}=0
$$

Apparently, $X=\left[x_{1}, x_{2}, x_{3}, \ldots, x_{D}, x_{D+1}\right]$ is the solution of the linear equations. By choosing $N$ years data, we can obtain an equation set containing $N$ equations with $(D+1)$ unknowns: $x_{1}-x_{D+1}$. Specific equations are as follows:

$$
\begin{gathered}
a_{11} x_{1}+a_{21} x_{2}+a_{31} x_{3}+\cdots+a_{D 1} x_{D}+x_{D+1}=0 \\
a_{12} x_{1}+a_{22} x_{2}+a_{32} x_{3}+\cdots+a_{D 2} x_{D}+x_{D+1}=0 \\
a_{13} x_{1}+a_{23} x_{2}+a_{33} x_{3}+\cdots+a_{D 3} x_{D}+x_{D+1}=0 \\
\vdots \\
a_{1 N} x_{1}+a_{2 N} x_{2}+a_{3 N} x_{3}+\cdots+a_{D N} x_{D}+x_{D+1}=0
\end{gathered}
$$

Similarly, this is equivalent to obtain the minimum of the equation below:

$$
\begin{aligned}
& \min F(A) \\
&=\sum_{N=1}^{N}\left[f\left(a_{1 N}, a_{2 N}, a_{3 N}, \ldots, a_{D N}\right)-\mathrm{Tec}_{N}\right]^{2}, \\
& \quad l \mathrm{~b} \leq a_{D N} \leq \mathrm{ub} .
\end{aligned}
$$

The subsequent procedure is using Artificial Bee Colony (ABC) algorithm to solve the multivariate linear equations and get coefficient $X=\left[x_{1}, x_{2}, x_{3}, \ldots, x_{D}, x_{D+1}\right]$. Then the relationship between the various factors and electricity consumption is explicit.

As a general rule, if an equation set has solutions, the number of independent variables $(D+1)$ should be greater than or equal to the number of equations $(N)$. Nevertheless, in this paper, $(D+1)$ will be less than $N$ and consequently it will result in no solution in this equation set. Under this circumstance, $\mathrm{ABC}$ algorithm cannot be used directly. Therefore, there are two improvements used in traditional ABC algorithm. To begin with, we apply multivariate linear regression (MLR) to $\mathrm{ABC}$ algorithm so that the no solution equation set can be solved. In other words, although the solution equation set is no solution, an optimal solution can be found when the error becomes the minimum. Secondly, we set a cycle index CYCLE2 which should be at least as big in order to avoid running into local optimum as much as possible. The concrete implementation steps are as follows.

Step 1 (initialization parameter). Set the population size $S N$, the maximum of iterative times CYCLE1, each iteration step size $V$, cycle index CYCLE2, the upper bound ub and lower bound $\mathrm{lb}$, data volume $N$, and the number of independent variables $D+1 ; \varepsilon$ is $F(A)$ and Lim is limit.

Step 2 (generate the initial solution). In the light of (3), it will generate $S N$ initial solutions randomly. Then according to (4) and (5), calculate the fitness and selected probability of each solution. At last choose the best fitness nectar source as the initial solution.

Step 3 (iterative optimization). Scouts search the neighborhood of the nectar source and calculate the fitness and the probability according to (4) and (5). If near honey is better than original nectar source, record new nectar source location and send to employed bees. Onlookers choose the nectar source according to $P_{i}$ and record new nectar source location according to (6). Then search the neighborhood of the new nectar source until there is no better one. At this moment, the number of iterations needs to be plus 1 .

Step 4 (limit judge). Save the current location of nectar source; if the number of iteration is beyond the maximum iterations CYCLE1, Step 2 is in return. Do like this CYCLE2 times.

Step 5 (find the optimal solution). Select the best solution from CYCLE2 nectar sources and it is perceived to be the optimal solution.

The program flow chart is shown in Figure 3.

This study forecasts the electricity consumption using an improved Artificial Bee Colony (ABC) algorithm to solve the multivariate linear equations. The data from 1990 to 2009, 20 years in the aggregate, is the basis and the data from 2010 to 2014,5 years, is used to test. So in this paper specific parameter is as follows: $S N=40$, CYCLE1 $=200, V=0.0001$, 


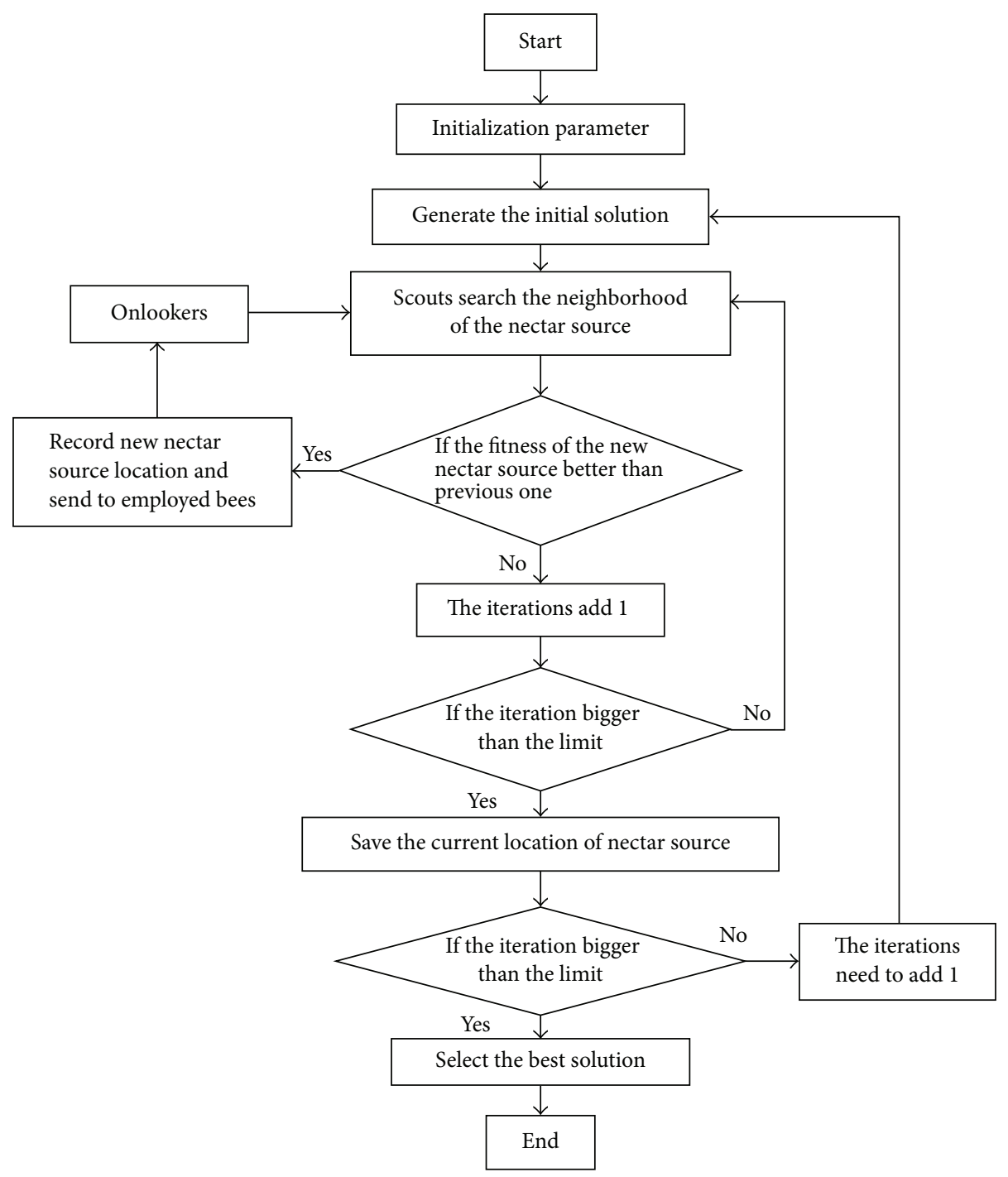

FIGURE 3: The program flow chart of the improved ABC model.

CYCLE2 $=1000, \mathrm{ub}=1$ and $\mathrm{lb}=-1, \operatorname{Lim}=0.01, D=8$, and $N$ $=20$. There are some explanations about the determination of parameters:

(1) Usually, the population size $S N$ is always 40 and there is no necessity to change in this paper.

(2) Because of $D+1=9$, as a computational algorithm, stochastic algorithm is a pseudorandom algorithm, so CYCLE1 $=200$ is enough to insure that every variable can be turned to when $D=8$.

(3) $V$ and CYCLE2 can improve the accuracy of the prediction. Under ideal conditions, $V$ should be as small as possible and CYCLE2 should be as big as possible. However, in consideration of the actual conditions, we valued $V=0.0001$ and CYCLE2 $=1000$.

(4) Because the data has been standardized, the coefficient can not be beyond $[-1,1]$. So $\mathrm{ub}=1$ and $\mathrm{lb}=$ -1 .
(5) Lim is a parameter which is used to control the end time. If $\operatorname{Lim}<0.01, \varepsilon$ is small enough for us to do prediction. So we valued $\operatorname{Lim}=0.01$.

To test whether the algorithm is correct or not, the following models are chosen to compare: (I) quadratic regression; (II) Artificial Neural Network (ANN); (III) Genetic Algorithm (GA); (IV) Particle Swarm Optimization (PSO).

As one of the most primitive prediction models, the quadratic regression mode should be selected to clarify whether this kind of models is still adaptive or not in such a complex circumstance. ANN algorithm is a representation of early artificial intelligence algorithm. It can tell us whether the emerging intelligence algorithm is better than the old one. GA and PSO are used to represent two classes of meta-heuristics separately and they will prove whether the improved ABC algorithm is superior to others. 


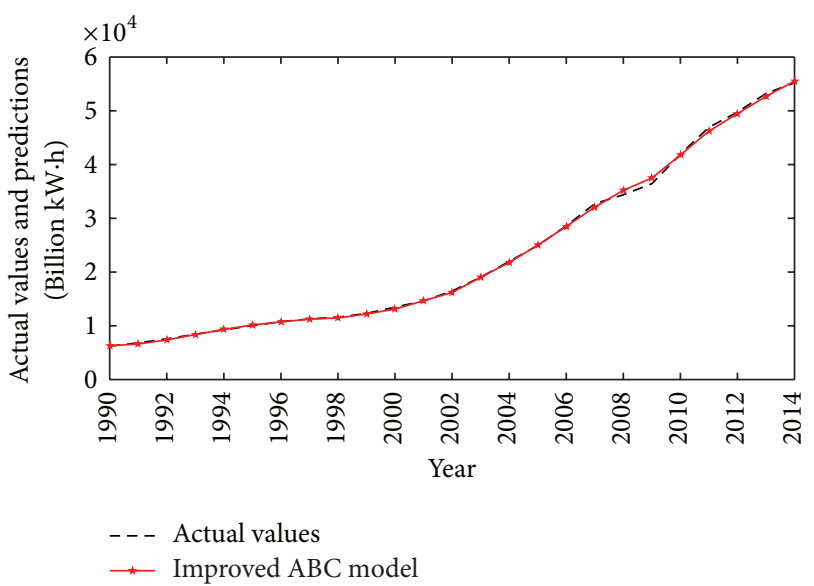

Figure 4: Numerical results of the improved ABC model.

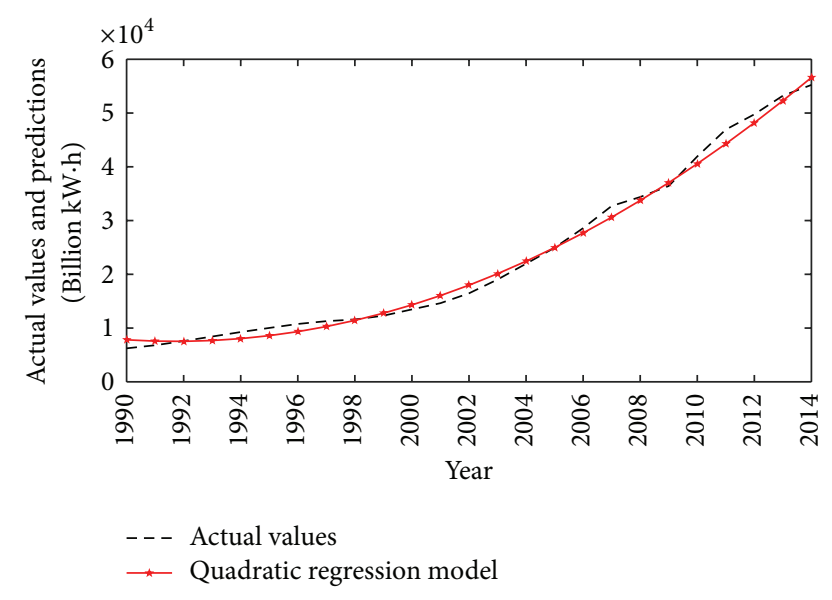

FIgURE 5: Numerical results of the quadratic regression model.

\section{Result and Discussion}

In this test, five algorithms run on matlab2015b and experimental results are shown in Figures 4-8.

Numerical results of the improved ABC model compared with actual values are shown in Figure 4.

Numerical results of the quadratic regression model compared with actual values are shown in Figure 5.

Numerical results of the ANN model compared with actual values are shown in Figure 6.

Numerical results of the GA model compared with actual values are shown in Figure 7.

Numerical results of the PSO model compared with actual values are shown in Figure 8.

Although there are figures of five models prediction results, we can not clearly see whether the results of electricity consumption based on the improved ABC algorithm are superior to the results based on other models only by our eyes. For the purpose of better reflection of differences, Table 4 was made and it provided precise predictions and the error between predictions and actual values. The performance of the proposed method is evaluated by two indices, namely, the mean absolute error (MAE) and the mean absolute

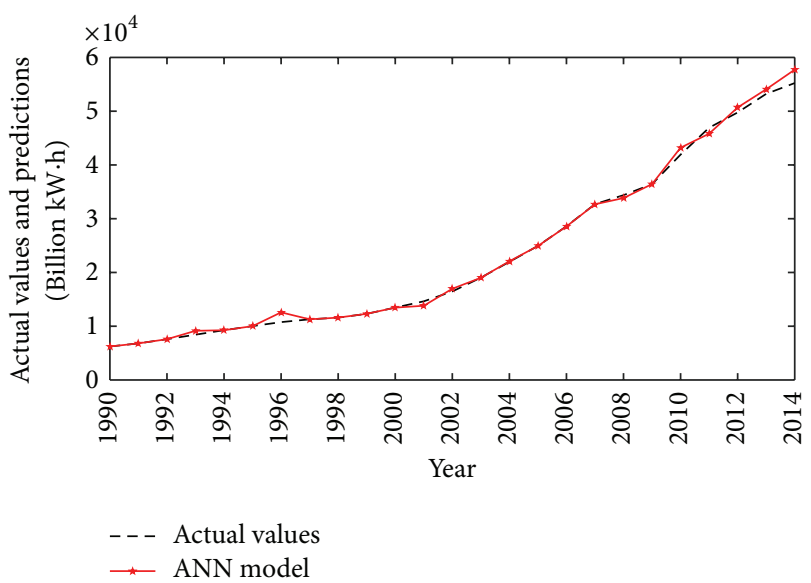

Figure 6: Numerical results of the ANN model.

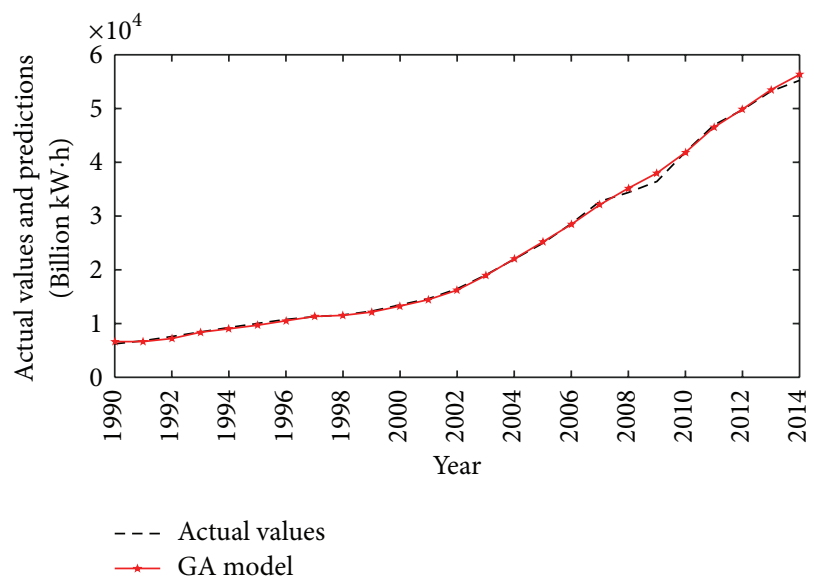

FIgURE 7: Numerical results of the GA model.

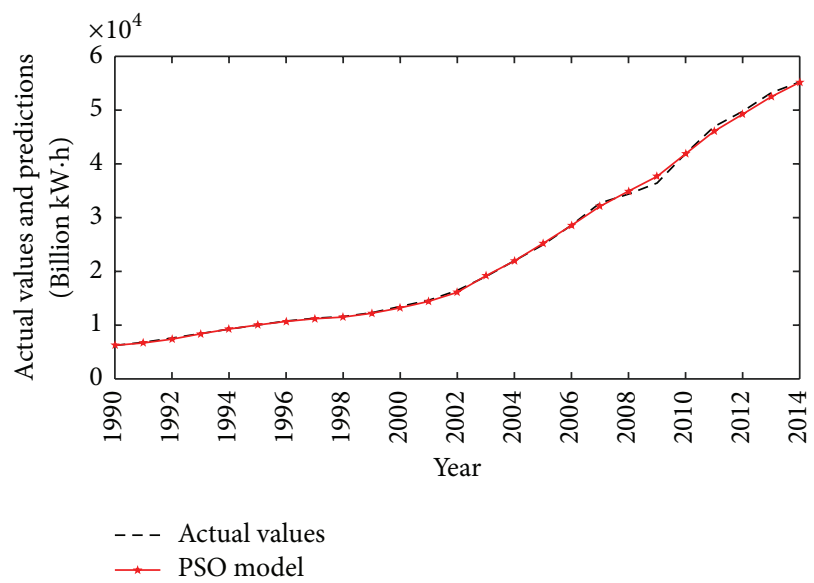

Figure 8: Numerical results of the PSO model.

percentage error (MAPE). The forecasting error of these models is shown in Table 4.

The forecasting error curves of these models are shown in Figure 9.

From error comparisons, the predicted effect of various methods can be clearly seen. 
TABLE 4: The forecasting error of these models.

\begin{tabular}{|c|c|c|c|c|c|c|c|}
\hline $\begin{array}{l}\text { Prediction } \\
\text { methods }\end{array}$ & Year & $\begin{array}{c}\text { TEC } \\
\text { (billion } \\
\mathrm{kW} \cdot \mathrm{h} \text { ) }\end{array}$ & $\begin{array}{c}\text { Predictions } \\
\text { (billion } \mathrm{kW} \cdot \mathrm{h} \text { ) }\end{array}$ & $\mathrm{AE}$ & APE & MAE & MAPE \\
\hline \multirow{5}{*}{$\begin{array}{l}\text { The improved } \mathrm{ABC} \\
\text { model }\end{array}$} & 2010 & 41923.00 & 41779.58 & 143.42 & $0.342 \%$ & \multirow{5}{*}{388.95} & \multirow{5}{*}{$0.928 \%$} \\
\hline & 2011 & 46928.00 & 46231.69 & 696.31 & $1.484 \%$ & & \\
\hline & 2012 & 49762.64 & 49455.53 & 307.11 & $0.617 \%$ & & \\
\hline & 2013 & 53223.00 & 52698.57 & 524.43 & $0.985 \%$ & & \\
\hline & 2014 & 55233.00 & 55506.47 & 273.47 & $0.495 \%$ & & \\
\hline \multirow{5}{*}{$\begin{array}{l}\text { The quadratic } \\
\text { regression mode }\end{array}$} & 2010 & 41923.00 & 40563.41 & 1359.59 & $3.243 \%$ & \multirow{5}{*}{1576.54} & \multirow{5}{*}{$3.761 \%$} \\
\hline & 2011 & 46928.00 & 44276.43 & 2651.57 & $5.650 \%$ & & \\
\hline & 2012 & 49762.64 & 48187.10 & 1575.54 & $3.166 \%$ & & \\
\hline & 2013 & 53223.00 & 52295.45 & 927.55 & $1.743 \%$ & & \\
\hline & 2014 & 55233.00 & 56601.46 & 1368.46 & $2.478 \%$ & & \\
\hline \multirow{5}{*}{ The ANN model } & 2010 & 41923.00 & 43212.85 & 1289.85 & $3.077 \%$ & \multirow{5}{*}{1335.66} & \multirow{5}{*}{$3.186 \%$} \\
\hline & 2011 & 46928.00 & 45814.30 & 1113.70 & $2.373 \%$ & & \\
\hline & 2012 & 49762.64 & 50693.92 & 931.28 & $1.871 \%$ & & \\
\hline & 2013 & 53223.00 & 54074.38 & 851.38 & $1.600 \%$ & & \\
\hline & 2014 & 55233.00 & 57725.06 & 2492.06 & $4.512 \%$ & & \\
\hline \multirow{5}{*}{ The GA model } & 2010 & 41838.90 & 41870.05 & 31.15 & $0.074 \%$ & \multirow{5}{*}{659.23} & \multirow{5}{*}{$1.576 \%$} \\
\hline & 2011 & 46503.54 & 46059.59 & 443.96 & $0.955 \%$ & & \\
\hline & 2012 & 49881.91 & 49236.22 & 645.69 & $1.294 \%$ & & \\
\hline & 2013 & 53493.77 & 52476.52 & 1017.25 & $1.902 \%$ & & \\
\hline & 2014 & 56330.92 & 55172.84 & 1158.08 & $2.056 \%$ & & \\
\hline \multirow{5}{*}{ The PSO model } & 2010 & 41923.00 & 41870.05 & 52.95 & $0.126 \%$ & \multirow{5}{*}{450.89} & \multirow{5}{*}{$1.076 \%$} \\
\hline & 2011 & 46928.00 & 46059.59 & 868.41 & $1.851 \%$ & & \\
\hline & 2012 & 49762.64 & 49236.22 & 526.42 & $1.058 \%$ & & \\
\hline & 2013 & 53223.00 & 52476.52 & 746.48 & $1.403 \%$ & & \\
\hline & 2014 & 55233.00 & 55172.84 & 60.16 & $0.109 \%$ & & \\
\hline
\end{tabular}

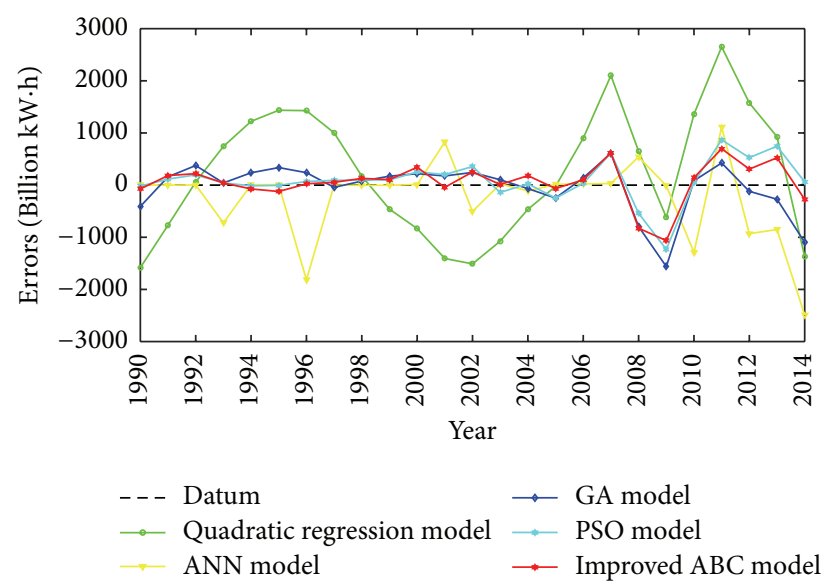

FIGURE 9: The forecasting errors curves of these models.

To begin with, the accuracy of quadratic regression mode is the worst. It is expected that the errors will be even bigger if the forecast period becomes longer. ANN model is better than quadratic regression mode at accuracy. However there is still a greater error from actual value. Maybe it due to the training data is too little. So quadratic regression mode and ANN model are not so appropriate to forecasting Chinese electricity consumption under this situation.

What is more, the GA model and PSO model make a progress in accuracy and the MAE and MAPE are also small enough to the actual application. However, this paper proved that the improved $\mathrm{ABC}$ algorithm combined with multivariate linear regression model is better than others in accuracy and it is more appropriate to forecast the electricity consumption in such a circumstance.

Thirdly, along with the running of these meta-heuristics algorithm, the error, namely, $\varepsilon_{i}(i=1,2,3, \ldots, 1000)$, will be created. Rank $\varepsilon_{i}$ in descending order is shown in Figure 10.

From Figure 10, it is obvious that the change of $\varepsilon$ becomes smaller and smaller and the solution corresponding to the $\min -\varepsilon$ is perceived to be the optimal solution. Although all these three models can find an optimal solution and their optimal solutions do not have much difference, we also can find the convergence speed of the improved $\mathrm{ABC}$ algorithm is faster than GA and PSO. So, in this paper, the improved $\mathrm{ABC}$ algorithm is proved to be the best model to forecasting Chinese electricity consumption. 


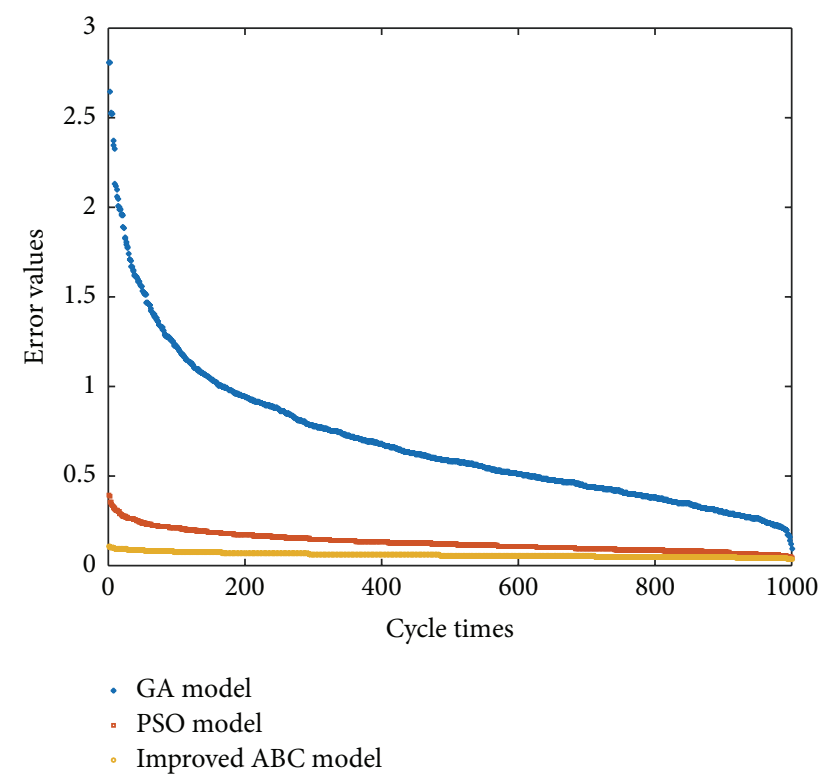

FIGURE 10: $\varepsilon_{i}$ in descending order of GA, PSP, and the improved ABC algorithm.

Finally, the optimal solution of the improved ABC algorithm, corresponding to the $\min -\varepsilon$, is $X=(0.385,0.434$, $0.584,0.486,0.375,0.722,0.265,0.052,0.883)$ Thereinto, 0.883 is the constant term. 0.052 is the coefficient of population. 0.722 is the coefficient of carbon emission. From the coefficients we can see that the population is unimportant influencing electricity consumption and there is close relation between carbon emission and electricity consumption. As for other factors, although they are not the most important factors, they should be taken into account.

Therefore, while forecasting the electricity consumption, it should not only focus on itself. The preferable way is to reference various factors associated with electricity consumption and find the relationship between them. It is reliable to get the electricity consumption through predicting the various factors' change in the future.

\section{Conclusions}

This paper suggests an improved $\mathrm{ABC}$ algorithm to explore the influencing mechanism of various factors on Chinese electricity consumption and forecast electricity consumption. Though arranging the research achievement, the historical situation of Chinese electricity use, and the change in demand of "new normal" electricity, eight factors are brought forward. By employing improved $\mathrm{ABC}$ and the eight factors, this paper investigates the influence mechanism of Chinese electricity consumption and builds a corresponding prediction model. Experiments proved that the model can well predict the Chinese electricity consumption in the future and have an advantage over simple ANN model and quadratic regression model in accuracy. It provides a new scientific and effective way to forecast the medium and long term electricity consumption.

\author{
Abbreviations \\ ABC: Artificial Bee Colony \\ MLR: Multivariate linear regression \\ ANN: Artificial Neural Network \\ GDP: Gross domestic product \\ GNP: Gross national product \\ CPI: Consumer price index \\ SVM: Support Vector Machine \\ FAI: Fixed asset investment \\ FDI: Foreign direct investment.
}

\section{Competing Interests}

The authors declare no conflict of interests.

\section{Authors' Contributions}

Professor Jingmin Wang planned the work. Under her guidance and encouragement, Jing Nie summarized the development of the research status and designed the model presented in this work. Jian Zhang implemented the main part of the different prediction methods and error analysis.

\section{Acknowledgments}

This work is part of Beijing Social and Scientific Fund which provides scientific supervision and guidance. The authors would like to express their acknowledgements to the Beijing Social and Scientific Fund for the financial support under 15JGB050.

\section{References}

[1] M.-X. Cui and J.-S. Wang, Annual Report on China's Energy Development (2013), vol. 1, Social Sciences Academic Press, Beijing, China, 2014.

[2] China National Renewable Energy Center, The Renewable Energy Industrial Development Report 2014, China Environmental Science Press, Beijing, China, 2014.

[3] E. Almeshaiei and H. Soltan, "A methodology for Electric Power Load Forecasting," Alexandria Engineering Journal, vol. 50, no. 2, pp. 137-144, 2011.

[4] M. J. OrtizBeviá, A. RuizdeElvira, and F. J. Alvarez-García, "The influence of meteorological variability on the mid-term evolution of the electricity load," Energy, vol. 76, pp. 850-856, 2014.

[5] W.-J. Lee and J. Hong, "A hybrid dynamic and fuzzy time series model for mid-term power load forecasting," International Journal of Electrical Power \& Energy Systems, vol. 64, pp. 10571062, 2015.

[6] V. Bianco, O. Manca, and S. Nardini, "Electricity consumption forecasting in Italy using linear regression models," Energy, vol. 34, no. 9, pp. 1413-1421, 2009.

[7] E. Erdogdu, "Electricity demand analysis using cointegration and ARIMA modelling: a case study of Turkey," Energy Policy, vol. 35, no. 2, pp. 1129-1146, 2007.

[8] Z. Dilaver Zafer and L. C. Hunt, "Industrial electricity demand for Turkey: a structural time series analysis," Energy Economics, vol. 33, no. 3, pp. 426-436, 2011. 
[9] Z. Dilaver and L. C. Hunt, "Modelling and forecasting Turkish residential electricity demand," Energy Policy, vol. 39, no. 6, pp. 3117-3127, 2011.

[10] D. Akay and M. Atak, "Grey prediction with rolling mechanism for electricity demand forecasting of Turkey," Energy, vol. 32, no. 9, pp. 1670-1675, 2007.

[11] H.-T. Pao, "Comparing linear and nonlinear forecasts for Taiwan's electricity consumption," Energy, vol. 31, no. 12, pp. 1793-1805, 2006.

[12] R. E. Kalman, "A new approach to linear filtering and prediction problems," Journal of Basic Engineering, vol. 82, no. 1, pp. 35-45, 1960.

[13] R. Inglesi, "Aggregate electricity demand in South Africa: conditional forecasts to 2030," Applied Energy, vol. 87, no. 1, pp. 197-204, 2010.

[14] H. Hahn, S. Meyer-Nieberg, and S. Pickl, "Electric load forecasting methods: tools for decision making," European Journal of Operational Research, vol. 199, no. 3, pp. 902-907, 2009.

[15] H. R. S. Keyno, F. Ghaderi, A. Azade, and J. Razmi, "Forecasting electricity consumption by clustering data in order to decline the periodic variable's affects and simplification the pattern," Energy Conversion and Management, vol. 50, no. 3, pp. 829-836, 2009.

[16] Y. Chen, G.-F. Zhang, T.-D. Jin, S.-M. Wu, and B. Peng, "Quantitative modelling of electricity consumption using computational intelligence aided design," Journal of Cleaner Production, vol. 69, pp. 143-152, 2014.

[17] F. Karanfil and Y.-J. Li, "Electricity consumption and economic growth: exploring panel-specific differences," Energy Policy, vol. 82, no. 1, pp. 264-277, 2015.

[18] A. Ciarreta and A. Zarraga, "Economic growth-electricity consumption causality in 12 European countries: a dynamic panel data approach," Energy Policy, vol. 38, no. 7, pp. 37903796, 2010.

[19] V. Bianco, O. Manca, and S. Nardini, "Linear regression models to forecast electricity consumption in Italy," Energy Sources, Part B: Economics, Planning and Policy, vol. 8, no. 1, pp. 86-93, 2013.

[20] C. F. Tang, M. Shahbaz, and M. Arouri, "Re-investigating the electricity consumption and economic growth nexus in portugal," Energy Policy, vol. 62, pp. 1515-1524, 2013.

[21] M. L. Polemis and A. S. Dagoumas, "The electricity consumption and economic growth nexus: evidence from Greece," Energy Policy, vol. 62, pp. 798-808, 2013.

[22] K. Zaman, M. M. Khan, M. Ahmad, and R. Rustam, "Determinants of electricity consumption function in Pakistan: old wine in a new bottle," Energy Policy, vol. 50, pp. 623-634, 2012.

[23] K. Kavaklioglu, "Modeling and prediction of Turkey's electricity consumption using support vector regression," Applied Energy, vol. 88, no. 1, pp. 368-375, 2011.

[24] B. Liddle and S. Lung, "Might electricity consumption cause urbanization instead? Evidence from heterogeneous panel longrun causality tests," Global Environmental Change, vol. 24, no. 1, pp. 42-51, 2014.

[25] S. A. Solarin and M. Shahbaz, "Trivariate causality between economic growth, urbanisation and electricity consumption in Angola: cointegration and causality analysis," Energy Policy, vol. 60, pp. 876-884, 2013.

[26] T. Zachariadis and N. Pashourtidou, "An empirical analysis of electricity consumption in Cyprus," Energy Economics, vol. 29, no. 2, pp. 183-198, 2007.
[27] J. Zhang, X.-Y. Yang, F. Shen et al., "Principal component analysis of electricity consumption factors in China," Energy Procedia, vol. 16, pp. 1913-1918, 2012.

[28] M. Meng and D. Niu, "Annual electricity consumption analysis and forecasting of China based on few observations methods," Energy Conversion and Management, vol. 52, no. 2, pp. 953-957, 2011.

[29] M. Shahbaz, R. Sbia, H. Hamdi, and I. Ozturk, "Economic growth, electricity consumption, urbanization and environmental degradation relationship in United Arab Emirates," Ecological Indicators, vol. 45, pp. 622-631, 2014.

[30] W. N. Cowan, T. Chang, R. Inglesi-Lotz, and R. Gupta, "The nexus of electricity consumption, economic growth and $\mathrm{CO}_{2}$ emissions in the BRICS countries," Energy Policy, vol. 66, pp. 359-368, 2014.

[31] L. Liu, X.-Q. Ma, and J.-L. Sun, "An investigation of the relationship between economic growth and electricity consumption with different industrial structures in different regions in China," in Proceedings of the 48th International Universities' Power Engineering Conference (UPEC '13), pp. 1-6, Dublin, Ireland, September 2013.

[32] W. Sun, C.-J. Lu, and M. Meng, "The application of optimal combined forecasting technique based on GRA in Mid/long term load forecasting," in Proceedings of the China International Conference on Electricity Distribution (CICED '06), Beijing, China, September 2006.

[33] M. Xing, S.-J. Yang, D.-X. Niu, and W. Sun, "Research on gray optimization combination power load forecasting based on multivariate exponential weighting," Power System Technology, vol. 29, pp. 8-11, 2005.

[34] P. Zhou, B. W. Ang, and K. L. Poh, "A trigonometric grey prediction approach to forecasting electricity demand," Energy, vol. 31, no. 14, pp. 2503-2511, 2006.

[35] Y. Ohtsuka, T. Oga, and K. Kakamu, "Forecasting electricity demand in Japan: a bayesian spatial autoregressive ARMA approach," Computational Statistics and Data Analysis, vol. 54, no. 11, pp. 2721-2735, 2010.

[36] R. Weron, Modeling and Forecasting Electricity Loads and Prices: A Statistical Approach, John Wiley \& Sons Ltd, Oxford, UK, 2006.

[37] P. Li, M. Li, and D.-C. Liu, "Power load forecasting based on improved regression," Power System Technology, vol. 30, pp. 99104, 2006.

[38] S. S. Pappas, L. Ekonomou, P. Karampelas et al., "Electricity demand load forecasting of the Hellenic power system using an ARMA model," Electric Power Systems Research, vol. 80, no. 3, pp. 256-264, 2010.

[39] X.-R. Song, L. Li, and L.-Q. Zhang, "Study on long-term power load forecasting," Computer Simulation, vol. 31, pp. 132-135, 2014.

[40] R. P. Lippmann, "An introduction to computing with neural nets," IEEE ASSP Magazine, vol. 4, no. 2, pp. 4-22, 1987.

[41] K. Metaxiotis, A. Kagiannas, D. Askounis, and J. Psarras, "Artificial intelligence in short term electric load forecasting: a state-of-the-art survey for the researcher," Energy Conversion and Management, vol. 44, no. 9, pp. 1525-1534, 2003.

[42] A. Azadeh, S. F. Ghaderi, and S. Tarverdian, "Electrical energy consumption estimation by genetic algorithm," in Proceedings of the International Symposium on Industrial Electronics (ISIE '06), vol. 7, pp. 395-398, Montreal, Canada, July 2006. 
[43] A. Azadeh, S. F. Ghaderi, S. Tarverdian, and M. Saberi, "Integration of artificial neural networks and genetic algorithm to predict electrical energy consumption," Applied Mathematics and Computation, vol. 186, no. 2, pp. 1731-1741, 2007.

[44] M. S. Kiran, E. Özceylan, M. Gündüz, and T. Paksoy, "Swarm intelligence approaches to estimate electricity energy demand in Turkey," Knowledge-Based Systems, vol. 36, pp. 93-103, 2012.

[45] A. Azadeh, M. Taghipour, S. M. Asadzadeh, and M. Abdollahi, "Artificial immune simulation for improved forecasting of electricity consumption with random variations," International Journal of Electrical Power \& Energy Systems, vol. 55, pp. 205224, 2014

[46] D. Karaboga, "An idea based on honey bee swarm for numerical optimization," Technical Report TR06, Erciyes University, Kayseri, Turkey, 2005.

[47] D. Karaboga and B. Basturk, "On the performance of artificial bee colony (ABC) algorithm," Applied Soft Computing, vol. 8, no. 1, pp. 687-697, 2008.

[48] N. Karaboga, "A new design method based on artificial bee colony algorithm for digital IIR filters," Journal of the Franklin Institute, vol. 346, no. 4, pp. 328-348, 2009.

[49] W. Szeto and Y. Jiang, "Hybrid artificial bee colony algorithm for transit network design," Transportation Research Record, vol. 2284, pp. 47-56, 2012.

[50] P. M. Pradhan, "Design of cognitive radio engine using artificial bee colony algorithm," in Proceedings of the International Conference on Energy, Automation and Signal (ICEAS '11), pp. 399-402, Odisha, India, December 2011.

[51] L. Chen, L. Zhang, and Y. Guo, "Blind image separation method based on artificial bee colony algorithm," Advanced Materials Research, vol. 468-471, pp. 583-586, 2012.

[52] D. Karaboga and B. Akay, "A comparative study of Artificial Bee Colony algorithm," Applied Mathematics and Computation, vol. 214, no. 1, pp. 108-132, 2009.

[53] B. Akay and D. Karaboga, "Parameter tuning for the artificial bee colony algorithm," in Proceedings of the 1st International Conference on Computation Collective Intelligence, pp. 608-619, Springer, Berlin, Germany, 2009.

[54] B. Li, "Research on WNN modeling for gold price forecasting based on improved Artificial Bee Colony algorithm," Computational Intelligence and Neuroscience, vol. 2014, Article ID 270658, 10 pages, 2014. 


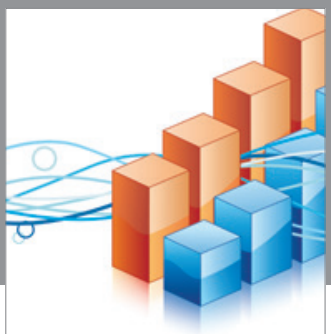

Advances in

Operations Research

vatem alat4

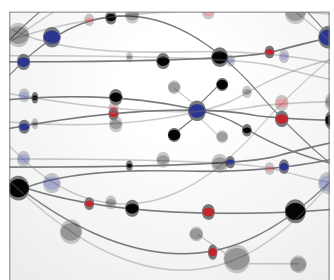

\section{The Scientific} World Journal
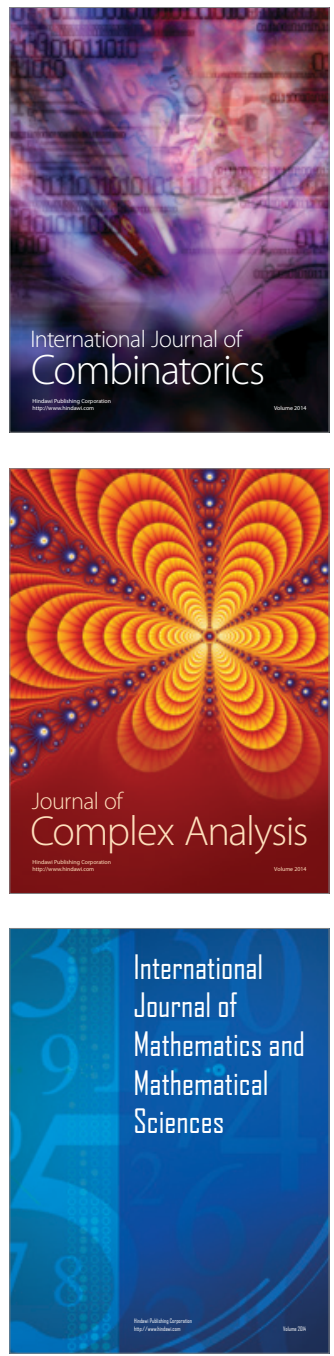
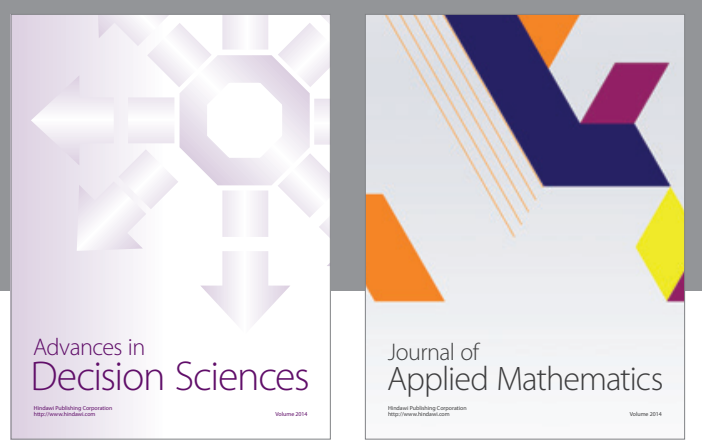

Algebra

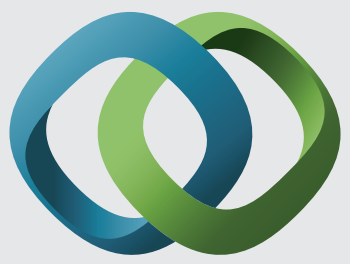

\section{Hindawi}

Submit your manuscripts at

http://www.hindawi.com
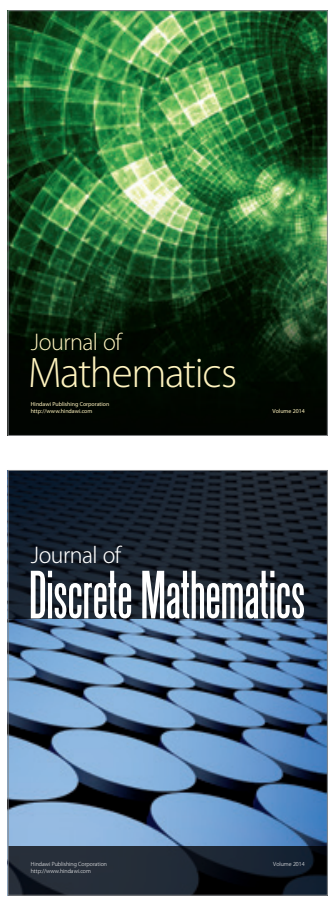

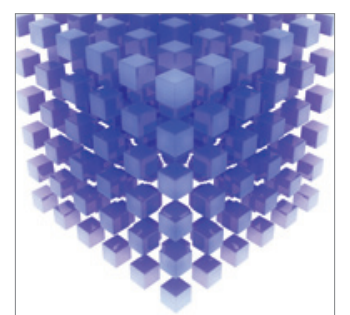

Mathematical Problems in Engineering
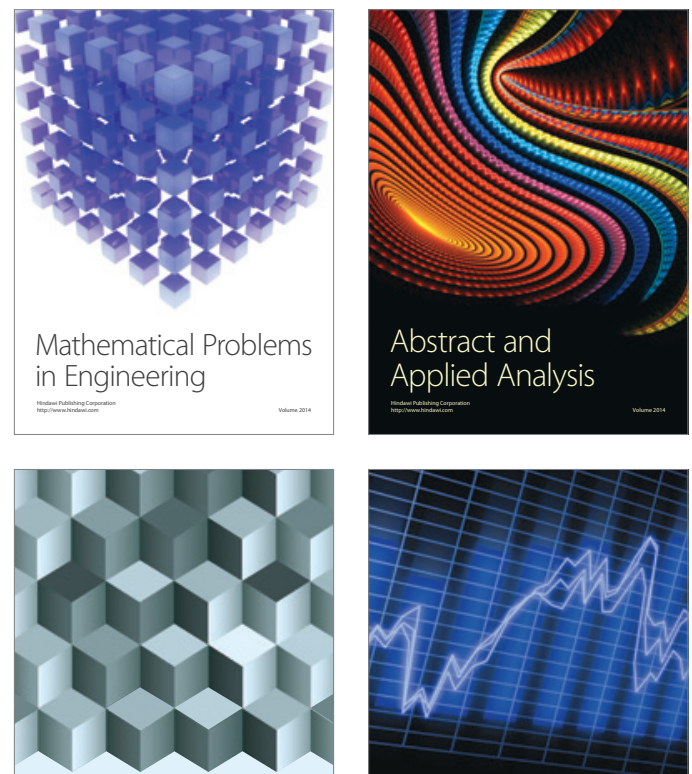

Journal of

Function Spaces

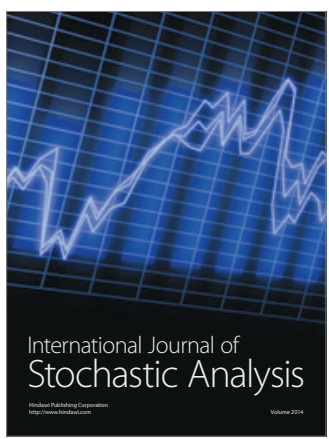

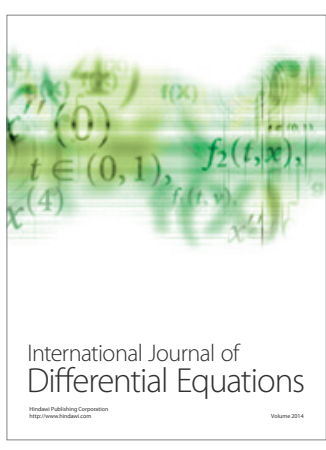
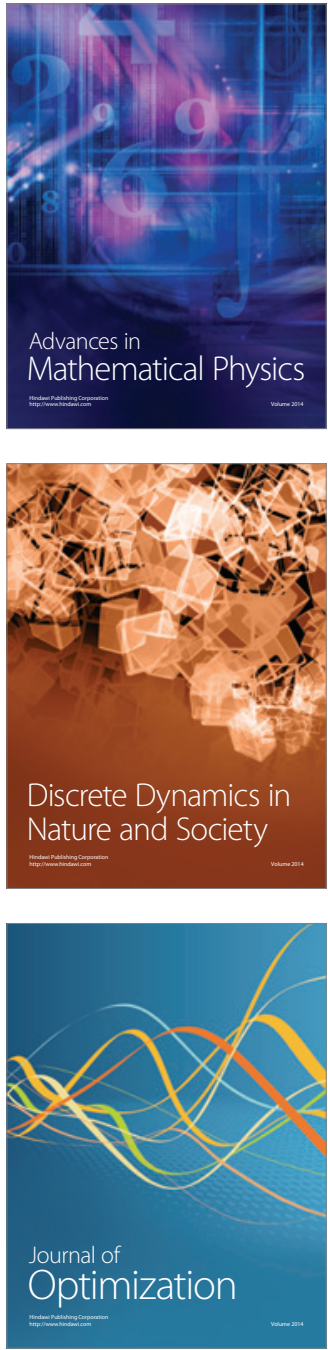